\title{
Predictive Performances of Blood Parameter Ratios for Liver Inflammation and Advanced Liver Fibrosis in Chronic Hepatitis B Infection
}

\author{
Rongrong Ding $\mathbb{D}^{1},{ }^{1}$ Xinlan Zhou, ${ }^{1}$ Dan Huang, ${ }^{1}$ Yanbing Wang, ${ }^{1}$ Xiufen Li, ${ }^{1}$ Li Yan, \\ Wei Lu, ${ }^{1}$ Zongguo Yang $\mathbb{D}^{2},{ }^{2}$ and Zhanqing Zhang ${ }^{1}{ }^{1}$ \\ ${ }^{1}$ Department of Hepatobiliary Medicine, Shanghai Public Health Clinical Center, Fudan University, Shanghai 201508, China \\ ${ }^{2}$ Department of Integrative Medicine, Shanghai Public Health Clinical Center, Fudan University, Shanghai 201508, China
}

Correspondence should be addressed to Zongguo Yang; yangzongguo@shphc.org.cn and Zhanqing Zhang; doctorzzqsphc@163.com

Received 4 December 2020; Revised 19 March 2021; Accepted 29 March 2021; Published 12 April 2021

Academic Editor: Washington L. C. dos Santos

Copyright $\odot 2021$ Rongrong Ding et al. This is an open access article distributed under the Creative Commons Attribution License, which permits unrestricted use, distribution, and reproduction in any medium, provided the original work is properly cited.

\begin{abstract}
Objective. Blood parameter ratios, including neutrophil to lymphocyte ratio (NLR), platelet to lymphocyte ratio (PLR), and monocyte to lymphocyte ratio (MLR), have been reported that they are correlated to the progression of liver disease. This study is aimed at evaluating the predictive value of PLR, NLR, and MLR for liver inflammation and fibrosis in patients with chronic hepatitis B (CHB). Methods. We recruited 457 patients with $\mathrm{CHB}$ who underwent a liver biopsy and routine laboratory tests. Liver histology was assessed according to the Scheuer scoring system. The predictive accuracy for liver inflammation and fibrosis was assessed by receiver operating characteristics (ROC) analysis. Results. PLR and NLR presented significantly reverse correlation to liver inflammation and fibrosis. However, these correlations were not observed for MLR and liver histology. The AUROCs of PLR for assessing G2-3 and G3 were 0.676 and 0.705 with cutoffs 74.27 and 68.75 , respectively. The AUROCs of NLR in predicting inflammatory scores G2-3 and G3 were 0.616 and 0.569 with cutoffs 1.36 and 1.85 , respectively. The AUROCs of PLR for evaluating fibrosis stages S3-4 and S4 were 0.723 and 0.757 with cutoffs 79.67 and 74.27 , respectively. The AUROCs of NLR for evaluating fibrosis stages S3-4 and S4 were 0.590 with cutoff 1.14. Conclusion. Although PLR has similar predictive power of progressive liver fibrosis compared with APRI, FIB-4, and GPR in CHB patients, it has the advantage of less cost and easy application with the potential to be widely used in clinical practice.
\end{abstract}

\section{Introduction}

Chronic hepatitis $\mathrm{B}(\mathrm{CHB})$ is still a serious public health problem worldwide that affects 257 million people all over the world [1]. It may cause progressive liver inflammation and fibrosis, cirrhosis, even end-stage liver disease, and hepatocellular carcinoma [2]. To reduce the burden of $\mathrm{CHB}$, the early and accurate prediction of liver inflammation and fibrosis as well as timely antiviral treatment plays an important role for controlling the disease progression, which even decreases the morbidity and mortality of CHB-related end-stage liver disease [3].
At present, liver biopsy is considered the gold standard procedure to accurately diagnose the liver histological scores. However, some limitations of liver biopsy restrict its widely clinical application such as invasiveness, patient's discomfort, sampling error, potential risk of complications, and interobserver variability $[4,5]$. In recent years, transient elasotgraphy (TE) has been introduced as a noninvasive, highly reproducible technique for assessment of liver fibrosis, especially in liver stages 3 and 4, which may reduce the need for liver biopsy [6-8]. Yet, some drawbacks such as expensive equipment and lack of trained operators limit the clinical application of TE especially in resource-limited 
environments. Therefore, many studies focus on developing simple and practical blood or serum noninvasive models, which are more accessible to the majority of the public [9].

WHO has recommended serum biomarkers including aspartate aminotransferase to the platelet ratio index (APRI) and four factor-based fibrosis index (FIB-4) as alternative methods for liver biopsy $[10,11]$. However, the performances of APRI and FIB-4 for evaluation of liver fibrosis are still controversial $[12,13]$. The gamma-glutamyl transpeptidase-toplatelet ratio (GPR) is more accurate than APRI and FIB-4 to estimate liver fibrosis in West Africa cohorts with $\mathrm{CHB}$, but it was not superior to APRI and FIB-4 in a French cohort [14]. Other studies also have not observed the advantages of GPR $[15,16]$.

Platelet-to-lymphocyte ratio (PLR), neutrophil-tolymphocyte ratio (NLR), and monocyte-to-lymphocyte ratio (MLR) are low-cost and easy to reproducible calculation even in simple laboratory conditions. Several researches have indicated that these ratios have values to be used as predictors for prognosis or inflammation in patients with cardiovascular disease, autoimmune diseases, and mood disorders [17-19]. Recently, Zhou et al. [20] reported that PLR and NLR were related to the disease severity in $\mathrm{CHB}$ patients. A study by Lu et al. [21] indicated that PLR could be useful in predicting liver advanced fibrosis and cirrhosis. Another recent study showed MLR and NLR may be potential prognostic markers for predicting poor outcome in patients with CHB-related liver failure. Nevertheless, the changes of these lymphocytes ratio models at different liver histological stages have been rarely studied. Therefore, we evaluated the clinical significances of the above six blood markers in predicting liver inflammation and fibrosis in $\mathrm{CHB}$ patients.

\section{Materials and Methods}

2.1. Ethic Statement. The study protocol and informed consent documents were reviewed and approved by the Ethics Committee of Shanghai Public Health Clinical Center, Fudan University. All these chronic hepatitis patients provided written informed consent before participating in this study.

2.2. Study Population. A total of 457 consecutive treatmentnaïve patients with $\mathrm{CHB}$ who underwent percutaneous liver biopsy at Shanghai Public Health Clinical Center, Fudan University, from December 2015 to January 2018 were retrospectively studied. The inclusion criteria were clinical history of $\mathrm{CHB}$ or HBsAg positive for more than 6 months, age $\geq$ 18 years, and discontinuation of potential lowering serum transaminase agents for at least 2 weeks prior to routine laboratory tests. The exclusion criteria were history of antiviral therapy, HCV and HIV coinfection, overt alcoholic or nonalcoholic fatty liver disease, autoimmune liver disease, hereditary metabolic liver diseases, decompensated cirrhosis, and pregnancy.

2.3. Liver Biopsy. Percutaneous liver biopsy was performed using a $16 \mathrm{G}$ needle under ultrasound guidance. Liver samples with a minimum length of $1.5 \mathrm{~cm}$ and at least 6 complete portal tracts were considered suitable for liver histological scoring $[22,23]$. Liver histology was analyzed by two experienced pathologists who were blinded to other clinical and laboratory data and classified according to the Scheuer scoring system [24].

2.4. Routine Laboratory Parameters. Fasting blood samples were obtained within a week of liver biopsy. Platelets and other blood cells were counted using a Sysmex-XT 4000i automated hematology analyzer. Serum alanine transaminase (ALT), aspartate aminotransferase (AST), alkaline phosphatase (ALP), gamma-glutamyl transfetase (GGT), bilirubin, albumin, and other serum biochemical parameters were measured using an Architectc16000 automatic biochemical analysis system. HBV DNA was quantified by real-time PCR (ABI 7500; Applied Biosystems, Foster City, CA, USA). Serum HBeAg was measured using a chemiluminescence microparticle immunoassay Abbot Architect 12000 automated analyzer and auxiliary reagents.

2.5. Formulas. The formulas for PLR, NLR, MLR, APRI, FIB4, and GPR are as follows: PLR = platelet count $\left(10^{9} / \mathrm{L}\right) /$ lymphocyte count $\left(10^{9} / \mathrm{L}\right), \quad \mathrm{NLR}=$ neutrophil count $\left(10^{9} / \mathrm{L}\right) /$ lymphocyte count $\left(10^{9} / \mathrm{L}\right), \quad \mathrm{MLR}=$ monocyte count $\left(10^{9} / \mathrm{L}\right) /$ lymphocyte count $\left(10^{9} / \mathrm{L}\right)$, APRI $=($ AST $(U / L) / U L N$ of AST )$/$ platelet count $\left(10^{9} / \mathrm{L}\right) \times 100, \mathrm{FIB}-4=($ age $($ years $) \times \mathrm{AST}$ $(\mathrm{U} / \mathrm{L})) /\left(\right.$ platelet count $\left.\left(10^{9} / \mathrm{L}\right) \times(\operatorname{ALT}(\mathrm{U} / \mathrm{L}))^{1 / 2}\right)$, and GPR $=(\mathrm{GGT}(\mathrm{U} / \mathrm{L}) / \mathrm{ULN}$ of GGT $) /$ platelet count $\left(10^{9} / \mathrm{L}\right) \times 100$.

2.6. Statistical Analysis. Statistical analysis was performed using IBM SPSS Statistics version 26.0 (SPSS Inc., Chicago, USA) and $R$ 4.0.4 (http://www.R-project.org). Continuous variables were given as the median (interquartile range, IQR) and compared using the independent Mann-Whitney test. Categorical variables were given as proportions and compared by the Chi-square test. Correlations were evaluated by Spear's correlation coefficient for continuous variables. The performances of serum models for predicting liver histological scores were assessed by receiver operating characteristic (ROC) curve analyses and the area under the ROC curves (AUROCs). The DeLong $Z$ test was used to compare the AUROC of the serum models. A two-sided $P<0.05$ was considered statistically significant difference.

\section{Results}

3.1. Patient Clinical Profiles. The baseline clinical characteristics of enrolled patients are described in Table 1 . The average age of the enrolled patients was 37 years. Most of them were men $(66.5 \%)$ and HBeAg positive (61.1\%). The distribution of liver inflammatory activities was 201 patients with G0-1 and 256 with G2-3, respectively. The distribution of fibrosis stages was 237 patients with S3-4 and 220 with S4, respectively. Compared with patients in G0-1, patients in G2-3 had higher ALT, AST, GGT, globulin, and HBV DNA, lower albumin, WBC counts, and platelet counts. Similarly, patients with S4 had higher ALT, AST, GGT, TBil, globin, APRI, FIB-4, and GPR, and lower albumin, platelet counts, PLR, and NLR. No significant differences were seen in MLR 


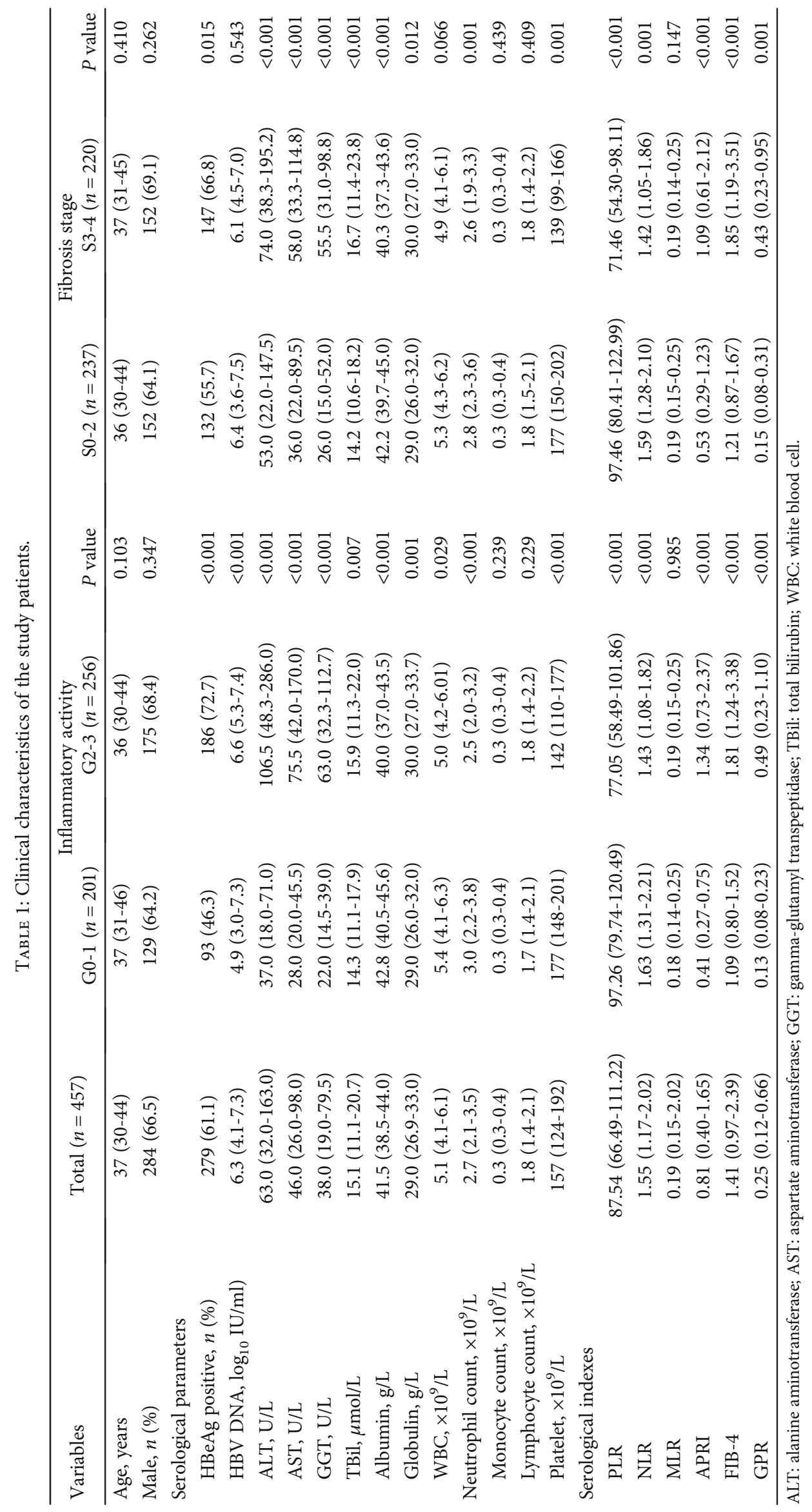




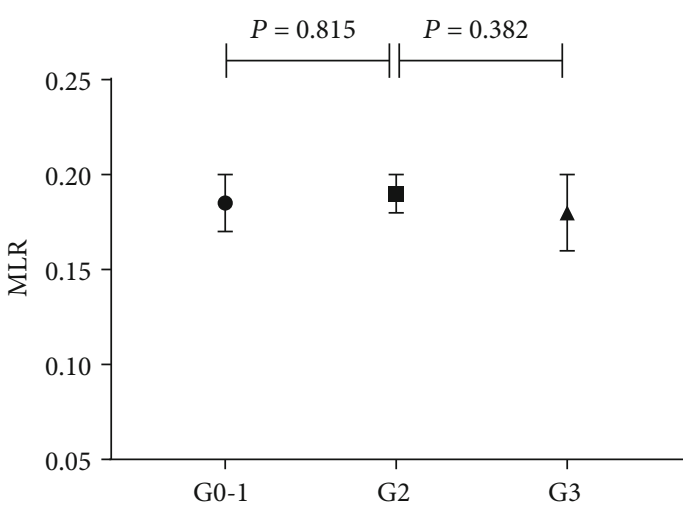

(a)

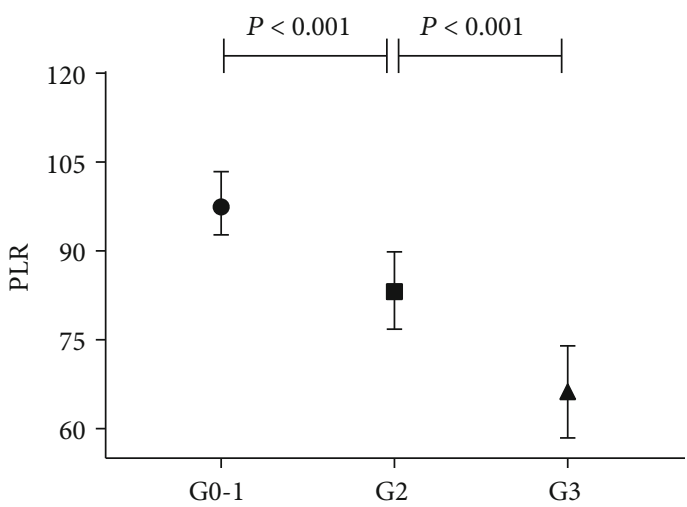

(c)

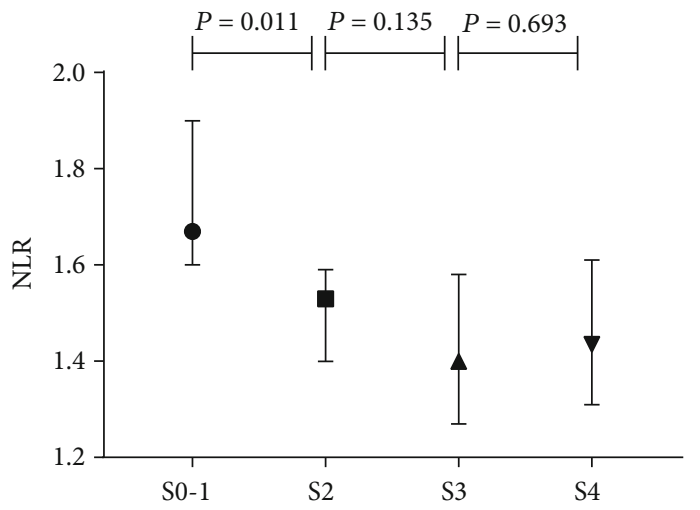

(e)

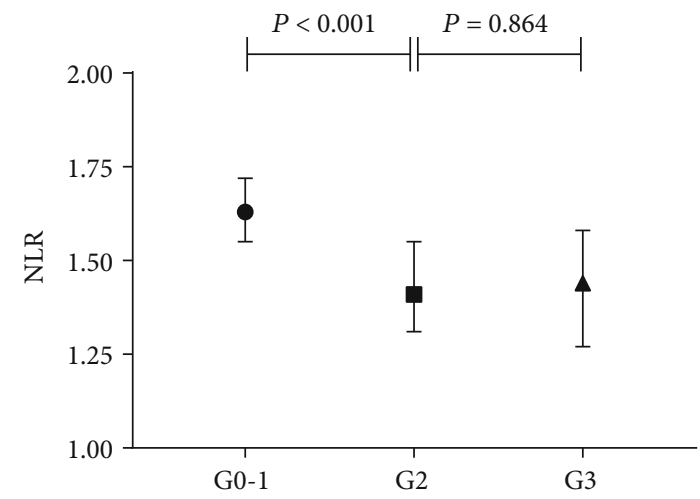

(b)

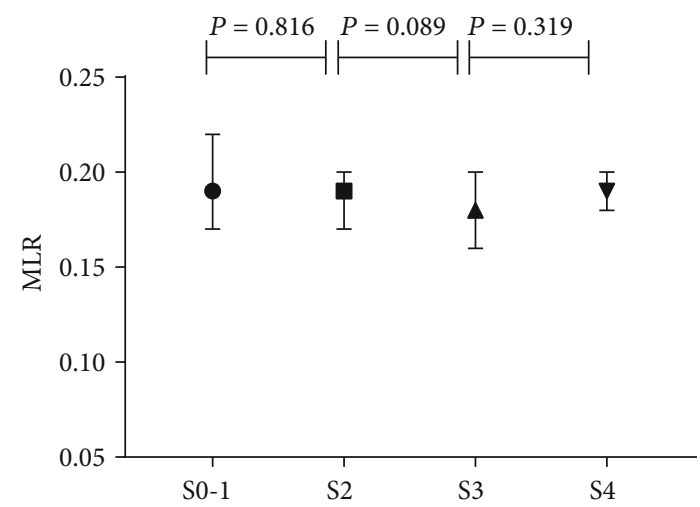

(d)

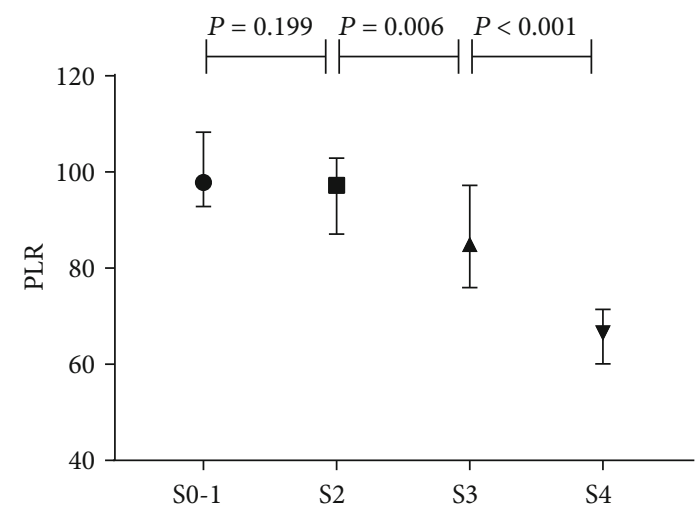

(f)

FIGURE 1: Medians in subgroups classified by inflammation grades and fibrosis stages (Scheuer scoring system). The medians of MLR (a) in G0-1, G2, and G3 were $0.18,0.19$, and 0.18 , respectively; the median of NLR (b) in G0-1, G2, and G3 were 1.63, 1.41, and 1.44, respectively, and the median of PLR (d) in G0-1, G2, and G3 were 97.46, 83.12, and 66.23, respectively. As for liver fibrosis, the medians of MLR (d) in S0-1, S2, S3, and S4 were 0.19, 0.19, 0.18, and 0.19, respectively; the medians of NLR (e) in S0-1, S2, S3, and S4 were 1.67, 1.53, 1.40, and 1.44, respectively, and the medians of PLR (f) in S0-1, S2, S3, and S4 were 97.85, 97.21, 84.97, and 66.50, respectively.

between patients with G0-1 and G2-3 or patients with S3-4 and S4.

3.2. Serological Models and Liver Histological Scores. The associations of PLR, NLR, and MLR with liver histopathology were further analyzed (Figures 1(a)-1(f)). As the liver histological scores increased, the PRL decreased. Spearman's correlation analysis presented that PLR $(r=-0.372)$, NLR $(r=-0.194)$, APRI $(r=0.586)$, FIB-4 $(r=0.470)$, and GPR $(r=0.601)$ were significantly correlated with liver inflammatory activities. As for liver fibrosis, PLR $(r=-0.414)$, NLR $(-0.172)$, APRI $(r=0.446)$, FIB-4 $(0.412)$, and GPR $(r=0.506)$ were significantly correlated with fibrosis stages (Table 2).

3.3. Performances of PLR, NLR, APRI, FIB-4, and GPR for the Evaluation of Liver Inflammation. The ROC curves of PLR, NLR, APRI, FIB-4, and GPR for predicting liver 
TABLE 2: Correlation between the noninvasive indexes and liver pathology score.

\begin{tabular}{lcccc}
\hline Indexes & \multicolumn{2}{c}{ Inflammatory activity } & \multicolumn{2}{c}{ Fibrosis stage } \\
& $r$ & $P$ value & $r$ & $<$ value \\
\hline PLR & -0.372 & $<0.001$ & -0.414 & $<0.001$ \\
NLR & -0.194 & $<0.001$ & -0.172 & $<0.001$ \\
MLR & -0.022 & 0.648 & -0.062 & 0.189 \\
APRI & 0.586 & $<0.001$ & 0.446 & $<0.001$ \\
FIB-4 & 0.470 & $<0.001$ & 0.412 & $<0.001$ \\
GPR & 0.601 & $<0.001$ & 0.506 & $<0.001$ \\
\hline
\end{tabular}

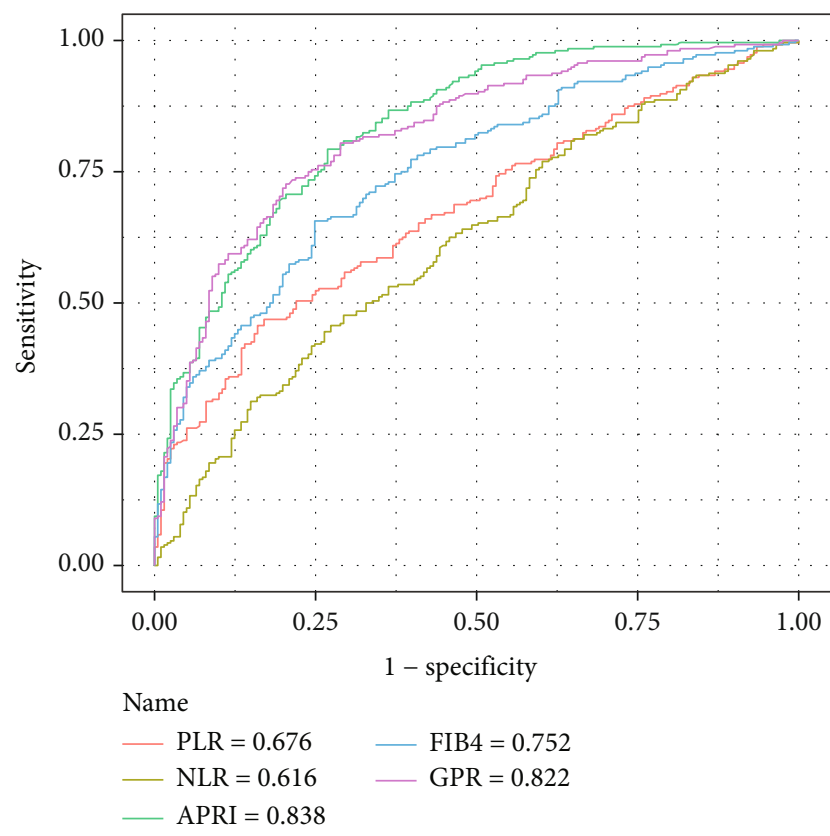

(a)

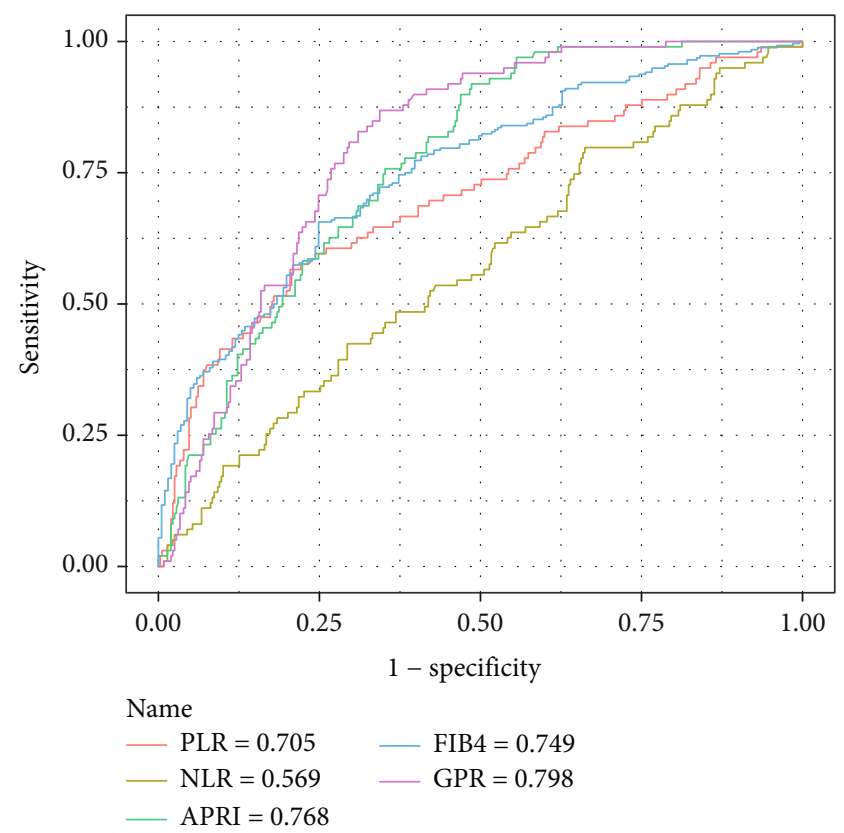

(b)

FIGURE 2: ROC comparison of PLR, NLR, APRI, FIB-4, and GPR for predicting liver inflammation. (a) ROC comparison for predicting G2-3. (b) ROC comparison for predicting G3.

inflammation in patients with $\mathrm{CHB}$ are shown in Figure 2. The diagnostic performances of the different markers are demonstrated in Table 3.

The AUROCs of PLR for assessing inflammatory scores G2-3 and G3 were $0.676(95 \% \mathrm{CI}=0.631-0.719)$ and 0.705 $(95 \% \mathrm{CI}=0.661-0.747)$ with cutoffs 74.27 and 68.75, respectively. The AUROCs of NLR in predicting inflammatory scores G2-3 and G3 were $0.616(95 \% \mathrm{CI}=0.570-0.661)$ and $0.569(95 \% \mathrm{CI}=0.523-0.615)$ with cutoffs 1.36 and 1.85 , respectively.

For the prediction of the inflammatory score G2-3, AUROC of PLR was better than that of NLR, but was significantly lower than APRI $(0.838,95 \% \mathrm{CI}=0.801-0.870, P<$ $0.0001)$, FIB-4 (0.752, 95\%CI $=0.710-0.791, P=0.009)$, and GPR $(0.822,95 \% \mathrm{CI}=0.784-0.856, P<0.0001)$. Regarding the prediction of the inflammatory score G3, although AUROC of PLR was still lower than GPR $(0.798,95 \% \mathrm{CI}=$ $0.759-0.834, P=0.009)$, it was superior to that of NLR and comparable with APRI $(0.768,95 \% \mathrm{CI}=0.727-0.806, P=$ $0.094)$ and FIB-4 $(0.749,95 \% \mathrm{CI}=0.706-0.788, P=0.269)$.
3.4. Performances of PLR, NLR, APRI, FIB-4, and GPR for the Evaluation of Liver Fibrosis. The ROC curves of PLR, NLR, APRI, FIB-4, and GPR for predicting liver fibrosis in patients with $\mathrm{CHB}$ are shown in Figure 3. The diagnostic performances of the different markers are demonstrated in Table 4.

The AUROCs of PLR for evaluating fibrosis stages S3-4 and S4 were $0.723(95 \% \mathrm{CI}=0.697-0.764)$ and 0.757 $(95 \% \mathrm{CI}=0.715-0.796)$ with cutoffs 79.67 and 74.27 , respectively. The AUROCs of NLR for evaluating fibrosis stages S3-4 and S4 were $0.590(95 \% \mathrm{CI}=0.544-0.636)$ with cutoff 1.14 . There was no statistically significant difference of the AUROC of NLR for staging S4.

For staging fibrosis S3-4, AUROC of PLR was higher than that of NLR and was comparable with APRI $(0.701,95$ $\% \mathrm{CI}=0.657-0.743, \quad P=0.448), \quad \mathrm{FIB}-4 \quad(0.697, \quad 95 \% \mathrm{CI}=$ $0.654-0.739, P=0.359)$, and GPR $(0.754,95 \% \mathrm{CI}=0.712$ $-0.793, P=0.272)$. Similarly, to stage S4, AUROC of PLR was comparable with APRI $(0.716,95 \% \mathrm{CI}=0.672-0.757$, $P=0.168)$, FIB-4 $(0.753,95 \% \mathrm{CI}=0.711-0.792, P=0.883)$, and GPR $(0.768,95 \% \mathrm{CI}=0.726-0.806, P=0.718)$. 
TABLE 3: Predictive performance of serological indexes for assessing liver inflammatory.

\begin{tabular}{|c|c|c|c|c|c|c|c|c|c|}
\hline & AUROC (95\% CI) & $P$ value & Cut-off & Se (\%) & Sp (\%) & PPV (\%) & NPV (\%) & Accuracy (\%) & ${ }^{*} P$ value \\
\hline \multicolumn{10}{|l|}{$\overline{P L R}$} \\
\hline $\mathrm{G} 2-3$ & $0.676(0.631-0.719)$ & $<0.0001$ & 74.27 & 46.9 & 83.0 & 54.2 & 78.5 & 62.6 & - \\
\hline G3 & $0.705(0.661-0.747)$ & $<0.0001$ & 68.75 & 56.6 & 79.6 & 54.2 & 83.9 & 72.9 & - \\
\hline \multicolumn{10}{|l|}{ NLR } \\
\hline G2-3 & $0.616(0.570-0.661)$ & $<0.0001$ & 1.36 & 45.7 & 72.6 & 41.7 & 75.7 & 57.3 & 0.044 \\
\hline G3 & $0.569(0.523-0.615)$ & 0.0337 & 1.85 & 79.8 & 33.8 & 34.0 & 79.5 & 57.8 & 0.0001 \\
\hline \multicolumn{10}{|l|}{ APRI } \\
\hline G2-3 & $0.838(0.801-0.870)$ & $<0.0001$ & 0.65 & 79.3 & 73.1 & 55.8 & 89.2 & 76.4 & $<0.0001$ \\
\hline G3 & $0.768(0.727-0.806)$ & $<0.0001$ & 0.68 & 91.9 & 51.4 & 44.8 & 93.7 & 60.6 & 0.094 \\
\hline \multicolumn{10}{|l|}{ FIB-4 } \\
\hline G2-3 & $0.752(0.710-0.791)$ & $<0.0001$ & 1.48 & 65.6 & 75.1 & 53.1 & 83.6 & 69.8 & 0.009 \\
\hline G3 & $0.749(0.706-0.788)$ & $<0.0001$ & 1.53 & 76.8 & 62.3 & 46.6 & 86.2 & 58.6 & 0.269 \\
\hline \multicolumn{10}{|l|}{ GPR } \\
\hline G2-3 & $0.822(0.784-0.856)$ & $<0.0001$ & 0.25 & 72.7 & 79.6 & 60.4 & 87.2 & 75.5 & $<0.0001$ \\
\hline G3 & $0.798(0.759-0.834)$ & $<0.0001$ & 0.29 & 86.9 & 65.6 & 52.0 & 92.1 & 70.7 & 0.009 \\
\hline
\end{tabular}

AUROC: area under ROC; Se: sensitivity; Sp: specificity; PPV: positive predictive value; NPV: negative predictive value. ${ }^{*}$ Compared with PLR.

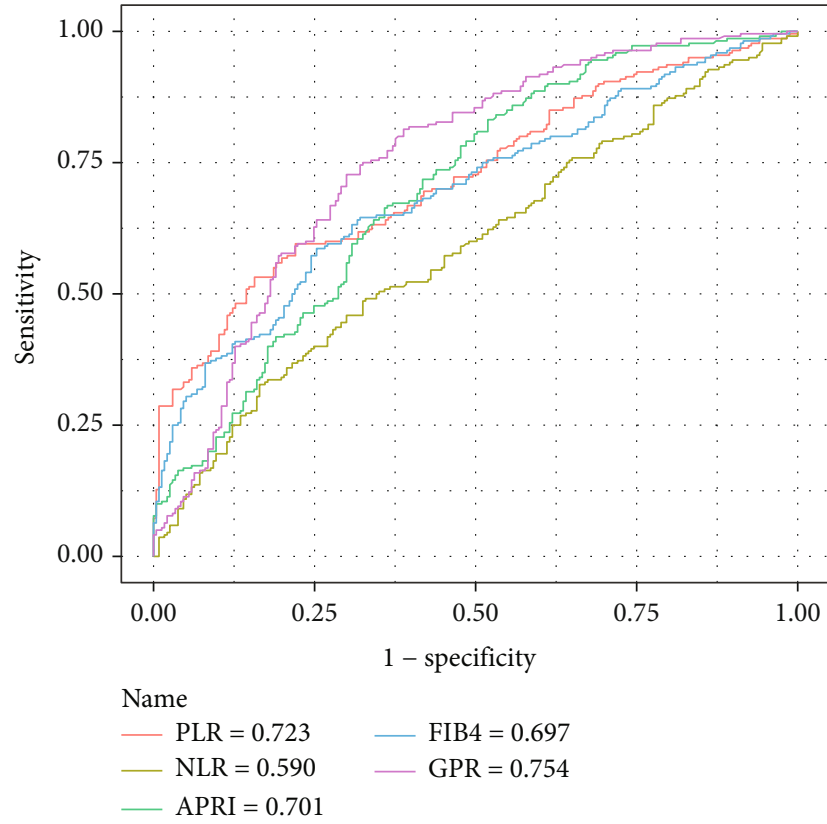

(a)

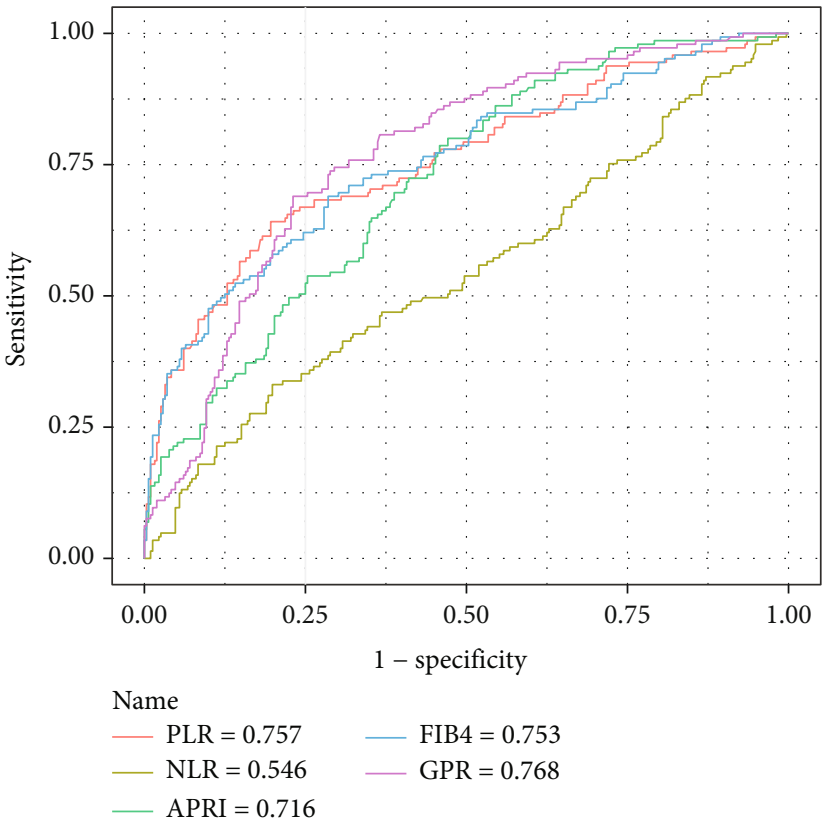

(b)

FIgURE 3: ROC comparison of PLR, NLR, APRI, FIB-4, and GPR for predicting liver fibrosis. (a) ROC comparison for predicting S3-4. (b) ROC comparison for predicting $\mathrm{S} 4$.

\section{Discussion}

Early diagnosis and accurate evaluation of liver inflammation and fibrosis are not only important for control of the progression of the disease but also for the treatment of chronic HBV infection [3]. Because of the limitations of liver biopsy, many researchers have tried to propose noninvasive ideal procedures to evaluate liver inflammation and fibrosis which should be simple, low-cost, repeatable, and accurate [13]. In the present study, we evaluated and compared the perfor- mances of PLR and NLR with APRI, FIB-4, and GPR, using histology as reference.

This study observed the presence of statistically significantly reverse correlations between the PLR values and liver pathological scores. PLR had good performance to stage advanced fibrosis (S3-4) with an AUROC of 0.72 at a cutoff of 9.67 and cirrhosis (S4) with an AUROC of 0.76 at a cutoff of 74.27. These results were consistent with previous study [21]. Lu et al. [21] reported the AUROC of PLR for advanced fibrosis was 0.7 at a cutoff of 73.27 and considered it as an 
TABle 4: Predictive performance of serological indexes for assessing liver fibrosis.

\begin{tabular}{|c|c|c|c|c|c|c|c|c|c|}
\hline & AUROC (95\% CI) & $P$ value & Cut-off & Se $(\%)$ & $\mathrm{Sp}(\%)$ & PPV (\%) & NPV (\%) & Accuracy & ${ }^{*} P$ value \\
\hline \multicolumn{10}{|l|}{ PLR } \\
\hline S3-4 & $0.723(0.697-0.764)$ & $<0.0001$ & 79.67 & 59.6 & 78.0 & 53.7 & 81.8 & 68.9 & - \\
\hline S4 & $0.757(0.715-0.796)$ & $<0.0001$ & 74.27 & 64.2 & 80.4 & 58.4 & 83.9 & 75.1 & - \\
\hline \multicolumn{10}{|l|}{ NLR } \\
\hline S3-4 & $0.590(0.544-0.636)$ & 0.0007 & 1.14 & 32.7 & 83.5 & 46.0 & 74.3 & 59.1 & $<0.0001$ \\
\hline S4 & $0.546(0.499-0.592)$ & 0.123 & - & - & - & - & - & - & - \\
\hline \multicolumn{10}{|l|}{ APRI } \\
\hline S3-4 & $0.701(0.657-0.743)$ & $<0.0001$ & 0.51 & 83.2 & 48.1 & 40.7 & 87.0 & 64.7 & 0.448 \\
\hline S4 & $0.716(0.672-0.757)$ & $<0.0001$ & 0.66 & 80.0 & 52.8 & 42.1 & 86.1 & 61.3 & 0.168 \\
\hline \multicolumn{10}{|l|}{ FIB-4 } \\
\hline S3-4 & $0.697(0.653-0.739)$ & $<0.0001$ & 1.61 & 58.6 & 74.7 & 49.8 & 80.8 & 66.5 & 0.359 \\
\hline S4 & $0.753(0.711-0.792)$ & $<0.0001$ & 1.65 & 70.0 & 71.5 & 50.9 & 84.3 & 69.6 & 0.883 \\
\hline \multicolumn{10}{|l|}{ GPR } \\
\hline S3-4 & $0.754(0.712-0.793)$ & $<0.0001$ & 0.25 & 72.7 & 70.0 & 51.0 & 85.7 & 70.2 & 0.272 \\
\hline S4 & $0.768(0.726-0.806)$ & $<0.0001$ & 0.38 & 69.0 & 76.9 & 56.2 & 85.3 & 74.0 & 0.718 \\
\hline
\end{tabular}

AUROC: area under ROC; Se: sensitivity; Sp: specificity; PPV: positive predictive value; NPV: negative predictive value. ${ }^{*}$ Compared with PLR.

easily available and cheap marker for evaluation of liver fibrosis and cirrhosis. Additionally, PLR performed comparably to classical serum biomarkers including APRI, FIB-4, and GPR. However, as for liver inflammation, the AUROCs of PLR for detecting significant inflammation (G2-3) and sever inflammation (G3) were 0.68 and 0.71 with cutoff at 74.27 and 68.75, respectively. In comparison with APRI and GPR, performance of PLR showed significantly lower AUROC for both assessment of G2-3 and G3. The PLR is a comprehensive indicator of changes in immune status during disease because it is calculated as the platelet count/lymphocyte count and accounts for variations in platelet and lymphocyte numbers [13]. The PLR value is also related to the progression and prognosis of sudden deafness, vestibular neuritis, cardiovascular disease, and thrombosis-related diseases [25-27]. Moreover, PLR values could serve as a predictor for the prognosis and progression of viral hepatitis and hepatocellular carcinoma [20, 28-30].

NLR also could be an important marker of systemic inflammation with the advantages including cost effect, ready availability, and easy calculation. It integrates two immune pathways that neutrophils indicate persistent inflammation and lymphocytes indicate the regulatory pathway [31]. NLR has been associated to various inflammations and cardiovascular diseases [17, 32, 33]. In addition, a higher NLR could predict poor prognosis in many cancers, hepatocellular, pancreatic, gastric cancers, and non-small-cell lung [34-37]. Recently, a few studies have estimated the predictive power of NLR in patients with liver fibrosis and liver cirrhosis. In our study, NLR also manifested a statistically significant reverse correlation with liver fibrosis. These observations were consistent with previous studies $[38,39]$. They showed that there was a possibility to use NLR as a predictive factor of liver fibrosis in $\mathrm{CHB}$ patients. However, in comparison with PLR, APRI, GPR, and FIB-4, performances of NLR to predict liver inflammation and fibrosis showed significantly lower AUROCs. Similar to our findings, a study by Huang et al. [40] demonstrated that the AUROCs of NLR for diagnosing advanced liver fibrosis and cirrhosis were 0.41 $(95 \% \mathrm{CI}=0.34-0.48)$ and $0.44(95 \% \mathrm{CI}=0.37-0.52)$. These results indicated that NLR did not sufficiently reflect the inflammation and the amount of the accumulated fibrous tissue in the liver.

Moreover, we validated the performance of APRI, GPR, and FIB-4 in diagnosing liver inflammation and fibrosis. The results showed that these classical noninvasive indexes were potential useful for diagnosis of liver inflammation and fibrosis. For liver inflammation, APRI and GPR were superior to FIB-4 for predicting G2-3, while the performances of the three markers were comparable for predicting G3. However, regarding liver fibrosis, GPR was superior to APRI and FIB-4. Similar to our study, a recent study by $\mathrm{Wu}$ et al. [12] showed that the AUROCs of APRI, FIB-4, and GPR for predicting $\geq$ G2 were $0.73,0.70,0.73$, and for $\mathrm{G} 3$ were $0.86,0.71$, and 0.88 , respectively. Another study showed lower AUROCs of the three markers for predicting liver inflammation that could be explained by the selection bias excluding $\mathrm{CHB}$ patients with the ALT level higher than two times of the ULN [41]. Additionally, our research and previous study confirmed that compared with APRI and FIB-4, GPR was more effective in evaluation of liver fibrosis $[11,14,42]$.

One limitation of this study was a single-centre retrospective study; thus, the results should be further confirmed in multicentre prospectively researches with large-scale populations. Furthermore, we could not evaluate the potential correlation between these markers with liver inflammation and fibrosis in patients with concomitant $\mathrm{CHB}$ and nonalcoholic fatty liver disease. It is reported that the prevalence of nonalcoholic fatty liver disease was $20 \%$ in patients with CHB [43].

In conclusion, the present study demonstrates that PLR is a potentially useful noninvasive marker for predicting 
advanced fibrosis and cirrhosis. Although PLR has similar predictive power of progressive liver fibrosis compare with APRI, FIB-4, and GPR in CHB patients, it has the advantage of less cost and easy application with the potential to be widely used in clinical practice. However, PRL does not show advantages in prediction of liver inflammation compared to APRI, FIB-4, and GPR.

\section{Data Availability}

Datasets of the current study are available from the corresponding authors on reasonable request.

\section{Conflicts of Interest}

The authors declare that there is no conflict of interests.

\section{Acknowledgments}

This work was supported by the "13th Five-year" National Science and Technology Major Project of China (2017ZX10203202).

\section{References}

[1] WHO, “Global Hepatitis Report; 2017," 2018 http://apps.who .int/iris/bitstream/10665/255017/255011/WHO-HIV-252017 .255006-eng.pdf.

[2] C. Trepo, H. L. Chan, and A. Lok, "Hepatitis B virus infection," Lancet, vol. 384, no. 9959, pp. 2053-2063, 2014.

[3] G. Shiha, A. Ibrahim, A. Helmy et al., "Asian-Pacific Association for the Study of the liver (APASL) consensus guidelines on invasive and non-invasive assessment of hepatic fibrosis: a 2016 update," Hepatology International, vol. 11, no. 1, pp. 130, 2017.

[4] The French METAVIR Cooperative Study Group and P. Bedossa, "Intraobserver and interobserver variations in liver biopsy interpretation in patients with chronic hepatitis C," Hepatology, vol. 20, no. 1, pp. 15-20, 1994.

[5] A. Regev, M. Berho, L. J. Jeffers et al., "Sampling error and intraobserver variation in liver biopsy in patients with chronic HCV infection," American Journal of Gastroenterology, vol. 97, no. 10, pp. 2614-2618, 2002.

[6] H. Dong, C. Xu, W. Zhou et al., "The combination of 5 serum markers compared to FibroScan to predict significant liver fibrosis in patients with chronic hepatitis B virus," Clinica Chimica Acta, vol. 483, pp. 145-150, 2018.

[7] R. Huang, N. Jiang, R. Yang et al., "Fibroscan improves the diagnosis sensitivity of liver fibrosis in patients with chronic hepatitis B," Experimental and Therapeutic Medicine, vol. 11, no. 5, pp. 1673-1677, 2016.

[8] Y. S. Seo, M. Y. Kim, S. U. Kim et al., "Accuracy of transient elastography in assessing liver fibrosis in chronic viral hepatitis: a multicentre, retrospective study," Liver International, vol. 35, no. 10, pp. 2246-2255, 2015.

[9] X. Q. Dong, Z. Wu, H. Zhao, G. Q. Wang, and China Hep BRFARG, "Evaluation and comparison of thirty noninvasive models for diagnosing liver fibrosis in chinese hepatitis B patients," Journal of Viral Hepatitis, vol. 26, no. 2, pp. 297307, 2019.
[10] World Health Organization, Guidelines for the Prevention, Care and Treatment of Persons with Chronic Hepatitis B Infection, World Health Organization, Geneva, 2015.

[11] M. Dong, J. Wu, X. Yu et al., "Validation and comparison of seventeen noninvasive models for evaluating liver fibrosis in Chinese hepatitis B patients," Liver International, vol. 38, no. 9, pp. 1562-1570, 2018.

[12] X. Wu, B. Cai, Z. Su et al., "Aspartate transaminase to platelet ratio index and gamma-glutamyl transpeptidase-to-platelet ratio outweigh fibrosis index based on four factors and red cell distribution width-platelet ratio in diagnosing liver fibrosis and inflammation in chronic hepatitis B," Journal of Clinical Laboratory Analysis, vol. 32, no. 4, article e22341, 2018.

[13] G. Xiao, J. Yang, and L. Yan, "Comparison of diagnostic accuracy of aspartate aminotransferase to platelet ratio index and fibrosis-4 index for detecting liver fibrosis in adult patients with chronic hepatitis B virus infection: a systemic review and meta-analysis," Hepatology, vol. 61, no. 1, pp. 292-302, 2015.

[14] M. Lemoine, Y. Shimakawa, S. Nayagam et al., "The gammaglutamyl transpeptidase to platelet ratio (GPR) predicts significant liver fibrosis and cirrhosis in patients with chronic HBV infection in West Africa," Gut, vol. 65, no. 8, pp. 1369-1376, 2016.

[15] Q. Li, W. Li, Y. Huang, and L. Chen, "The gamma-glutamyl transpeptidase-to-platelet ratio predicts liver fibrosis and cirrhosis in HBeAg-positive chronic HBV infection patients with high HBV DNA and normal or mildly elevated alanine transaminase levels in China," Journal of Viral Hepatitis, vol. 23, no. 11, pp. 912-919, 2016.

[16] W. Zhang, M. Sun, G. Chen et al., "Reassessment of gammaglutamyl transpeptidase to platelet ratio (GPR): a large-sample, dynamic study based on liver biopsy in a Chinese population with chronic hepatitis B virus (HBV) infection," Gut, vol. 67, no. 5, pp. 989-991, 2018.

[17] M. E. Afari and T. Bhat, "Neutrophil to lymphocyte ratio (NLR) and cardiovascular diseases: an update," Expert Review of Cardiovascular Therapy, vol. 14, no. 5, pp. 573-577, 2016.

[18] M. G. Mazza, S. Lucchi, A. G. M. Tringali, A. Rossetti, E. R. Botti, and M. Clerici, "Neutrophil/lymphocyte ratio and platelet/lymphocyte ratio in mood disorders: a meta-analysis," Progress in Neuro-Psychopharmacology and Biological Psychiatry, vol. 84, pp. 229-236, 2018.

[19] Z. Yang, Z. Zhang, F. Lin et al., "Comparisons of neutrophil-, monocyte-, eosinophil-, and basophil- lymphocyte ratios among various systemic autoimmune rheumatic diseases," APMIS, vol. 125, no. 10, pp. 863-871, 2017.

[20] Z. Zhao, J. Liu, J. Wang et al., "Platelet-to-lymphocyte ratio (PLR) and neutrophil-to-lymphocyte ratio (NLR) are associated with chronic hepatitis B virus (HBV) infection," International Immunopharmacology, vol. 51, pp. 1-8, 2017.

[21] W. Lu, Y. P. Zhang, H. G. Zhu et al., "Evaluation and comparison of the diagnostic performance of routine blood tests in predicting liver fibrosis in chronic hepatitis B infection," British Journal of Biomedical Science, vol. 76, no. 3, pp. 137-142, 2019.

[22] European Association for Study of L, Asociacion Latinoamericana para el Estudio del H, "EASL-ALEH clinical practice guidelines: non-invasive tests for evaluation of liver disease severity and prognosis," Journal of Hepatology, vol. 63, no. 1, pp. 237-264, 2015. 
[23] R. R. Ding, J. M. Zheng, D. Huang et al., "INR-to-platelet ratio (INPR) as a novel noninvasive index for predicting liver fibrosis in chronic hepatitis B," International Journal of Medical Sciences, vol. 18, no. 5, pp. 1159-1166, 2021.

[24] P. J. Scheuer, "Classification of chronic viral hepatitis: a need for reassessment," Journal of Hepatology, vol. 13, no. 3, pp. 372-374, 1991.

[25] H. Acet, F. Ertas, M. A. Akil et al., "Novel predictors of infarctrelated artery patency for ST-segment elevation myocardial infarction: Platelet-to-lymphocyte ratio, uric acid, and neutrophil-to-lymphocyte ratio," Anatolian Journal of Cardiology, vol. 15, no. 8, pp. 648-656, 2015.

[26] S. Shushan, S. Shemesh, O. J. Ungar et al., "Neutrophil-tolymphocyte ratio and platelet-to-lymphocyte ratio among patients with vestibular neuritis," ORL; Journal for Otorhino-laryngology and its Related Specialties, vol. 81, no. 5-6, pp. 304-308, 2019.

[27] W. Yang and Y. Liu, "Platelet-lymphocyte ratio is a predictor of venous thromboembolism in cancer patients," Thrombosis Research, vol. 136, no. 2, pp. 212-215, 2015.

[28] X. Li, L. Wang, and P. Gao, "Chronic hepatitis C virus infection: relationships between inflammatory marker levels and compensated liver cirrhosis," Medicine (Baltimore), vol. 98, no. 39, article e17300, 2019.

[29] A. Suner, B. I. Carr, H. Akkiz et al., "Inflammatory markers Creactive protein and PLR in relation to HCC characteristics," Journal of Translational Science, vol. 5, no. 3, p. 10, 2019.

[30] D. Wang, N. Bai, X. Hu et al., "Preoperative inflammatory markers of NLR and PLR as indicators of poor prognosis in resectable HCC," Peer J, vol. 7, article e 7132, p. e7132, 2019.

[31] P. Avanzas, J. Quiles, E. L. de Sá et al., "Neutrophil count and infarct size in patients with acute myocardial infarction," International Journal of Cardiology, vol. 97, no. 1, pp. 155156, 2004.

[32] T. Angkananard, T. Anothaisintawee, M. McEvoy, J. Attia, and A. Thakkinstian, "Neutrophil lymphocyte ratio and cardiovascular disease risk: a systematic review and meta-analysis," Biomed Reseach International, vol. 2018, article 2703518, 2018.

[33] H. Bhutta, R. Agha, J. Wong, T. Y. Tang, Y. G. Wilson, and S. R. Walsh, "Neutrophil-lymphocyte ratio predicts mediumterm survival following elective major vascular surgery: a cross-sectional study," Vascular and Endovascular Surgery, vol. 45, no. 3, pp. 227-231, 2011.

[34] J. Bruix, A. L. Cheng, G. Meinhard, K. Nakajima, Y. De Sanctis, and J. Llovet, "Prognostic factors and predictors of sorafenib benefit in patients with hepatocellular carcinoma: analysis of two phase III studies," Journal of Hepatology, vol. 67, no. 5, pp. 999-1008, 2017.

[35] T. Asaoka, A. Miyamoto, S. Maeda et al., "Prognostic impact of preoperative NLR and CA19-9 in pancreatic cancer," Pancreatology, vol. 16, no. 3, pp. 434-440, 2016.

[36] S. Diem, S. Schmid, M. Krapf et al., "Neutrophil-to-Lymphocyte ratio (NLR) and Platelet-to-Lymphocyte ratio (PLR) as prognostic markers in patients with non-small cell lung cancer (NSCLC) treated with nivolumab," Lung Cancer, vol. 111, pp. 176-181, 2017.

[37] R. Miyamoto, S. Inagawa, N. Sano, S. Tadano, S. Adachi, and M. Yamamoto, "The neutrophil-to-lymphocyte ratio (NLR) predicts short-term and long-term outcomes in gastric cancer patients," European Journal of Surgical Oncology, vol. 44, no. 5, pp. 607-612, 2018.
[38] A. Pokora Rodak, S. Kiciak, and K. Tomasiewicz, "Neutrophillymphocyte ratio and mean platelet volume as predictive factors for liver fibrosis and steatosis in patients with chronic hepatitis B," Annals of Agricultural and Environmental Medicine, vol. 25, no. 4, pp. 690-692, 2018.

[39] B. Yilmaz, H. Aydin, G. Can et al., "The relationship between fibrosis level and blood neutrophil to lymphocyte ratio in inactive hepatitis B carriers," European Journal of Gastroenterology Hepatology, vol. 26, no. 12, pp. 1325-1328, 2014.

[40] R. Huang, G. Wang, C. Tian et al., "Gamma-glutamyl-transpeptidase to platelet ratio is not superior to APRI,FIB-4 and RPR for diagnosing liver fibrosis in CHB patients in China," Scientific Reports, vol. 7, no. 1, p. 8543, 2017.

[41] L. Wang, J. Li, K. Yang et al., "Comparison and evaluation of non-invasive models in predicting liver inflammation and fibrosis of chronic hepatitis B virus-infected patients with high hepatitis B virus DNA and normal or mildly elevated alanine transaminase levels," Medicine (Baltimore), vol. 99, no. 23, article e20548, 2020.

[42] D. P. Liu, W. Lu, Z. Q. Zhang et al., "Comparative evaluation of GPR versus APRI and FIB-4 in predicting different levels of liver fibrosis of chronic hepatitis B," Journal of Viral Hepattitis, vol. 25, no. 5, pp. 581-589, 2018.

[43] S. Bondini, J. Kallman, A. Wheeler et al., "Impact of nonalcoholic fatty liver disease on chronic hepatitis B," Liver International, vol. 27, no. 5, pp. 607-611, 2007. 